\title{
Use of $\mathrm{MgSO} 4$ as alternative contrast medium in computed tomography scanning and analyzed by voltammetric technique
}

\author{
Muhammed Mizher Radhi \\ Health and Medical Technology College, Baghdad, Iraq
}

\begin{abstract}
Magnesium sulfate $\left(\mathrm{MgSO}_{4}\right)$ solution has been chosen as alternative contrast medium in computed tomography scanning (CT-scan). The solution of $\mathrm{MgSO}_{4}$ was a good electrochemical property especially in the blood medium. It was found that $\mathrm{MgSO}_{4}$ in blood medium served as an antioxidant reagent because cyclic voltammogram had only one reduction current peak at $0.9 \mathrm{~V}$, while iodine compounds were used as a common contrast medium in CT-scan; they had two oxidation current peaks in the blood medium at $0.73 \mathrm{~V}$ and $0.23 \mathrm{~V}$. In addition, the iodine compounds caused many problems when used as a contrast medium, but they were the only contrast media used for this purpose. It was found that using $\mathrm{MgSO}_{4}$ solution as an alternative contrast medium in rabbits enhanced CT-scan in the resolution and increasing Hounsfield unit (HF) values of kidney and heart organ, as compared with that using iodine compound at the same dose.
\end{abstract}

Keywords: $\mathrm{MgSO}_{4}$, contrast medium of CT-scanning, cyclic voltammetry, rabbit blood sample, Hounsfield unit

\section{INTRODUCTION}

Scientists have recently been interested in important studies to look for alternatives to the contrast media used in medical examination devices such as X-ray, CT-scan, and MRI that are safer than the currently used contrast media [1,2].

Some X-rays and a CT-scan (helix) may require the use of contrast media, depending on the type of examination and the disease that will be diagnosed. The contrast medium is a liquid substance that mostly contains iodine. The advantage of contrast in computerized tomography is that it increases the clarity of tissues, organs, and blood vessels. This increase in contrast is important in some diseases, and without the contrast medium the radiologist may not be able to distinguish some diseases [3]. Intravenous contrast media - The reason for calling it here is that this contrast is injected into a vein. The goal of venous variation is to improve and en- hance image clarity, especially for blood vessels, brain, liver, kidneys, and heart. It is transparent like water; the amount of contrast used is usually between 75 and $150 \mathrm{ml}$. The amount of variance granted to a patient depends on several factors, including: age, weight and type of organ to be diagnosed [4].

There are two factors in computerized tomography that may raise concerns about patient safety by taking an iodine contrast medium: the first factor, the body's sensitivity to the iodine compound before taking contrast and asking the patient about his medical history. Another is the problem of kidney failure: therefore, a kidney function test is performed before a CT scan [5]. There are different kind of iodine compounds which used as contrast media in the CT-scan examination: the first one is renal-oriented with highly-osmolarity e.g. diatrizoate, iodamide. The other one is renal orientation, with low osmolarity e.g. iohexol metramid, iopamidol [6]. 
An English salt or magnesium sulfate is an inorganic compound (chemical compound) containing magnesium, sulfur and oxygen, from the formula $\mathrm{MgSO} 4$. Usually called Epsom salt, English salt is a natural conditioner that helps treat constipation when ingested internally. It helps increase the water content inside the intestine. It is considered an intestinal antiseptic, so using $\mathrm{MgSO} 4$ as an alternative to contrast media in computed tomography is safety contrast [7]. Diagnosis with a CT-scan makes routine use of iodine contrast factors. Patients with kidney disease or contrast sensitivity impose restrictions on the use of iodine contrast. In such cases, alternative contrast media can be used to perform the procedure. Current alternatives to iodine include carbon dioxide, gadolinium and the diluted iodine contrast. Each of these alternatives has its unique advantages and limitations. Current iodine alternatives are explored, focusing on applications and their limitations $[8,9]$. Venous radial contrast is used for many different diagnostic procedures to improve images in different radiology studies. Examples of studies include CT-scans, angiography and pyelography. These diagnostic procedures are performed on a daily basis in radiology and interventional cardiac departments worldwide. In general, it is used to improve vascular vision [10].

In the current study, an important thing in the medical field of radiology, which the use of compounds that are dangerous to human health and used as a contrast media in diagnosis by radiology technique since they were discovered and used widely in this field. The alternative of these compounds was used of $\mathrm{MgSO} 4$ solution in X-ray and CT-scan as safety contrast media.

\section{EXPERIMENTAL}

\section{Materials}

Bayer Pharma AG Company from the German company (Berlin Germany) iodine contrast as Iopromide (Ultravist 370) was used as contrast media in $\mathrm{CT}$-scan. Magnesium sulfate $\left(\mathrm{MgSO}_{4}\right)$ was used from Chinese SCRC (China). Ascorbic acid (AA) was from Technicon Chemicals Co. (Oreq. Tournai Belgium). Anesthesia materials used to anesthetize animals such as ketamine 10\% from Alfasan company (Netherlands), xylazine 2\% from Alfasan (Netherlands). Blood samples of rabbits, and other chemicals and solvents were of annular grade and were used as received by the manufacturers. Deionized water was used to prepare aqueous solutions. All solutions were deaerated oxygen by nitrogen gas for 10-15 minutes prior to making measurement.

\section{Preparation magnesium sulfate $3 \mathrm{M} \mathrm{MgSO}_{4}$}

A 3 molar solution of pure magnesium sulfate (Chinese SCRC) was prepared in a $10 \mathrm{ml}$ volumetric flask, and the crystals were dissolved in deionized water to obtain a 3 molar solution of magnesium sulfate.

Cyclic voltammetry

Ezstat series (potentiostat/galvanostat) NuVant Systems Inc. pioneering electrochemical technologies USA.

Pyrex cell measuring 10 milliliters and three electrodes was used:

a. Working electrode: where the glass carbon electrode (GCE) was used

b. Reference electrode: where $\mathrm{Ag} / \mathrm{AgCl}$ silver electrode $(3 \mathrm{M} \mathrm{KCl})$ was used

c. Counter electrode: Where to use a platinum wire ( $1 \mathrm{~mm}$ diameter)

All three electrodes were dipped in the solution under study and linked to the potentiostat, which in turn was connected with the personal computer to identify the properties of the materials studied in the blood medium. The glassy carbon electrode (GCE) was used in this study after cleaning with alumina grand and sonic technique for 10-15 min.

\section{X-ray and CT-scan apparatuses}

The Toshiba-X-ray-Generater model DC12MC-2 was used by the CT-scan screw type Toshiba-Toshiba Scanner Aquilion-model TSX-101A.

After preparing the rabbit for examination and in the case of anesthesia with the specified dose of the contrast, the rabbit was lying on the examination table to perform the X-ray imaging process as shown in Figure 1 and the spiral CT-scan as shown in Figure 2.

\section{RESULTS AND DISCUSSION}

\section{Voltammetric effect of magnesium sulfate in the blood medium}

Magnesium sulfate $\left(\mathrm{MgSO}_{4}\right)$, the alternative contrast medium, was used in CT-scan, it was studied using an electrochemical method by cyclic 


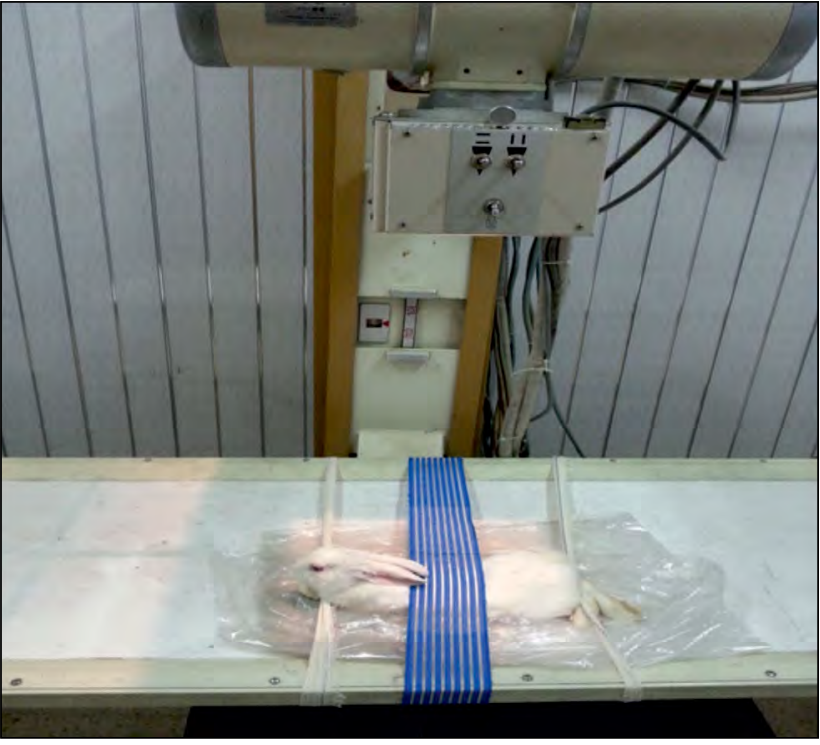

FIGURE 1. Preparation of the rabbit in X-ray

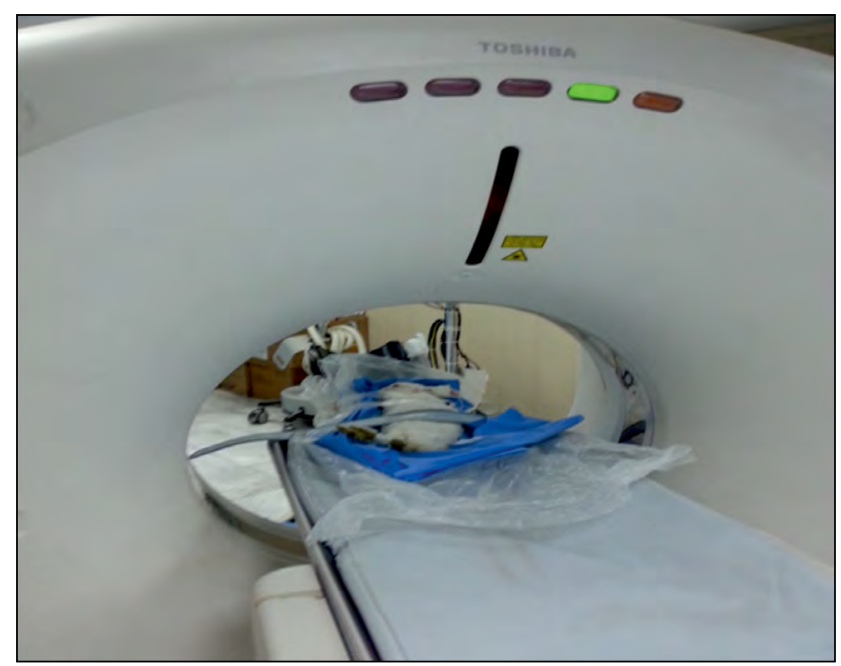

FIGURE 2. Preparation pf the rabbit in CT-scan voltammetry. Magnesium sulfate solution has good electrochemical properties, especially in the blood medium. It has been found that $\mathrm{Mg}$ (II) in the blood acts as an antioxidative reagent as shown in Figure 3 illustrated the appearance of two reduction current peaks of $\mathrm{Mg}(\mathrm{II})$ in two potential regions at -0.5 and $-1 \mathrm{~V}$, so $\mathrm{MgSO}_{4}$ solution can be considered as anti-oxidant reagent because it received the free radical in the electrochemical reaction with blood composition. So, it can be used $\mathrm{MgSO}_{4}$ solution as alternative contrast medium in safety behavior in blood medium [11].

\section{Electrochemical study of iodine compound in the blood medium}

One of the iodine compounds was used as one of the famous and only contrast medium used in images using X-ray technology and spiral CT-scan at the present time. As it showed the electrochemical properties in the blood medium as oxidative reagent, it showed several oxidative peaks in the blood and enhanced the two oxidation current peaks by increasing the concentration of iodine compound added to the blood, as shown in Figure 4. It can be concluded from this important analysis that all the compounds that contain iodine is harmful oxidizing substances through its interaction with the blood composition, which causes undesirable symptoms when taken intravenously for all patients who are undergoing diagnostic with both X-ray techniques

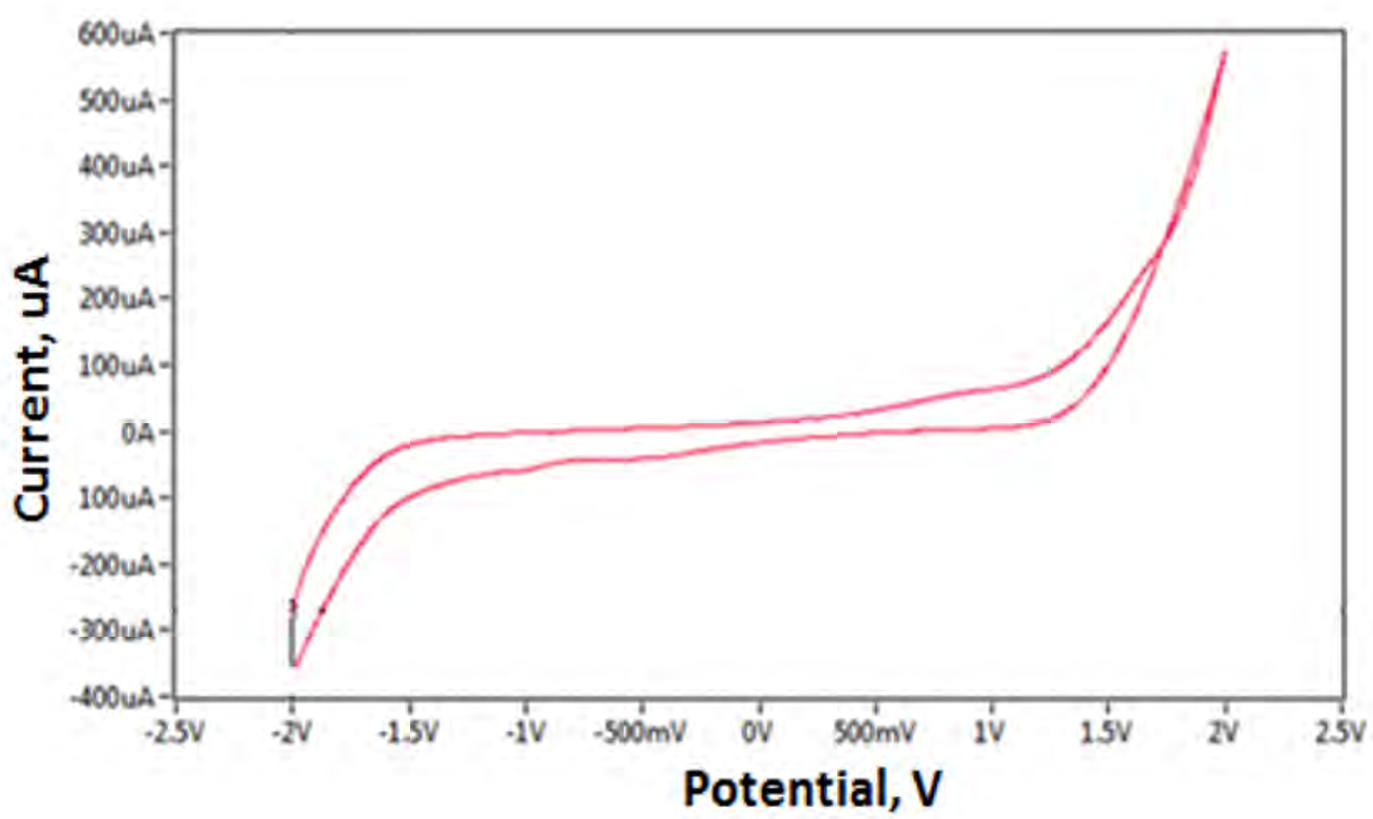

FIGURE 3. Cyclic voltammogram of $3 \mathrm{M} \mathrm{MgSO}_{4}$ as alternative contrast medium in blood 


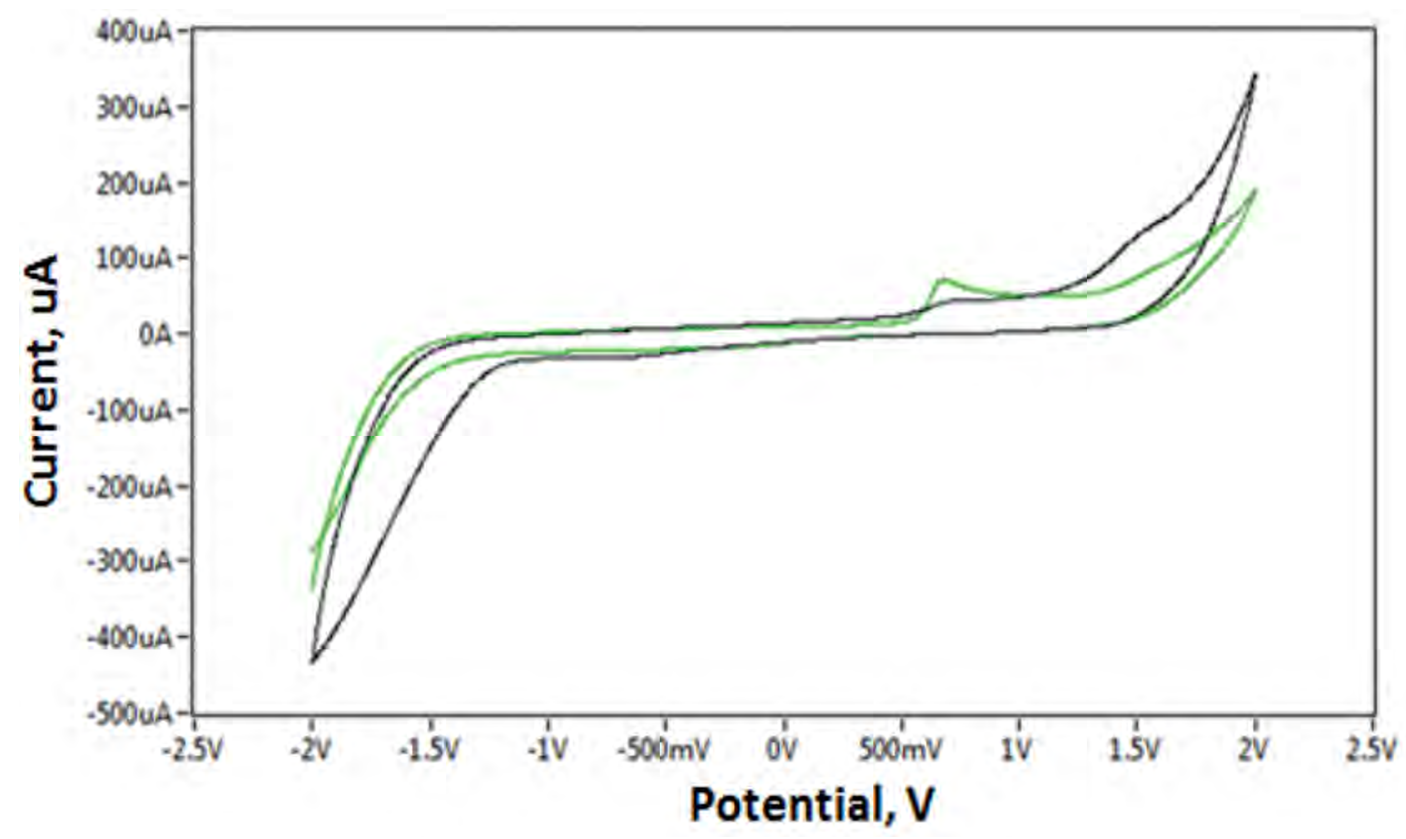

FIGURE 4. Cyclic voltammogram of iodine solution at different concentrations in blood medium

and CT-scan, in addition to the dangerous symptoms they cause for those who suffer from kidney or liver deficiencies or heart diseases must not be given the iodine contrast media to avoid complications that may cause death in certain cases or permanent diseases and at the same time it is forbidden to use the diagnosis required for this technique [12-14].

\section{Study the rabbits by CT-scan}

The rabbits were chosen to perform the CT-scan procedure for examinations in various organs of the rabbit's abdomen, especially the kidneys and bladder, as well as the heart, as follows.

The first group is the group that studied rabbit by CT-scan without using contrast media (Pre).

The second group, in which spatial scanning of rabbits was studied using the alternative contrast medium of magnesium sulfate solution, where the kidneys, heart and bladder of rabbits were studied by intravenously the alternative contrast of magnesium sulfate at a concentration of 3 molar at different doses ( $2 \mathrm{ml}$ and $4 \mathrm{ml}$ and $6 \mathrm{ml})$ and the following tests were performed using the spiral CT-scan.

\section{CT-scan examinations}

This examination was taken for all members of the abdominal area of the body of the rabbits such as kidney, bladder and heart without using any contrast media, then the iodine contrast was used and the examination was taken for all organs in the rabbits. The Hounsfield unit (HU) factor values can be used to determine the clarity of the CT-scan image.

Hounsfield unit (HU):

Absorption coefficient unit of radiolucency of a substance;

$\mathrm{HU}$ is normalized to water, where water $=0 \mathrm{HU}$, air $=-1000 \mathrm{HU}$ and bone $=1000 \mathrm{HU}$

The HU values in the CT-scan are reported for each case taken for the studied rabbits as follows:

1. The heart

Tests were taken to turn the rabbits into the following three cases:

A. Check the heart without using the contrast medium.

Note in Figure 5 of the rabbit's heart without using any contrast medium, and the value of the clarity of the heart of the HU was 40.2 where it is unclear.

B. Cardiac examination using an iodine contrast medium.

Heart in Figure 6 can be observed using the iodine contrast medium and the value of heart clearness $(\mathrm{HU}=104.5)$ where diagnosis is possible.

C. Cardiac examination using alternative contrast medium of magnesium sulfate solution. Figure 7 clearly shows the shape of the heart when using the heart's magnesium sulfate contrast medium and the value of clarity is high as it is easy to diagnose $(\mathrm{HU}=121.1)$. 
It can be seen from the three pictures that the highest clarity value was when using the alternative contrast medium of magnesium sulfate, where its value was 121.1 of $\mathrm{HU}$, which is the highest value among the values.

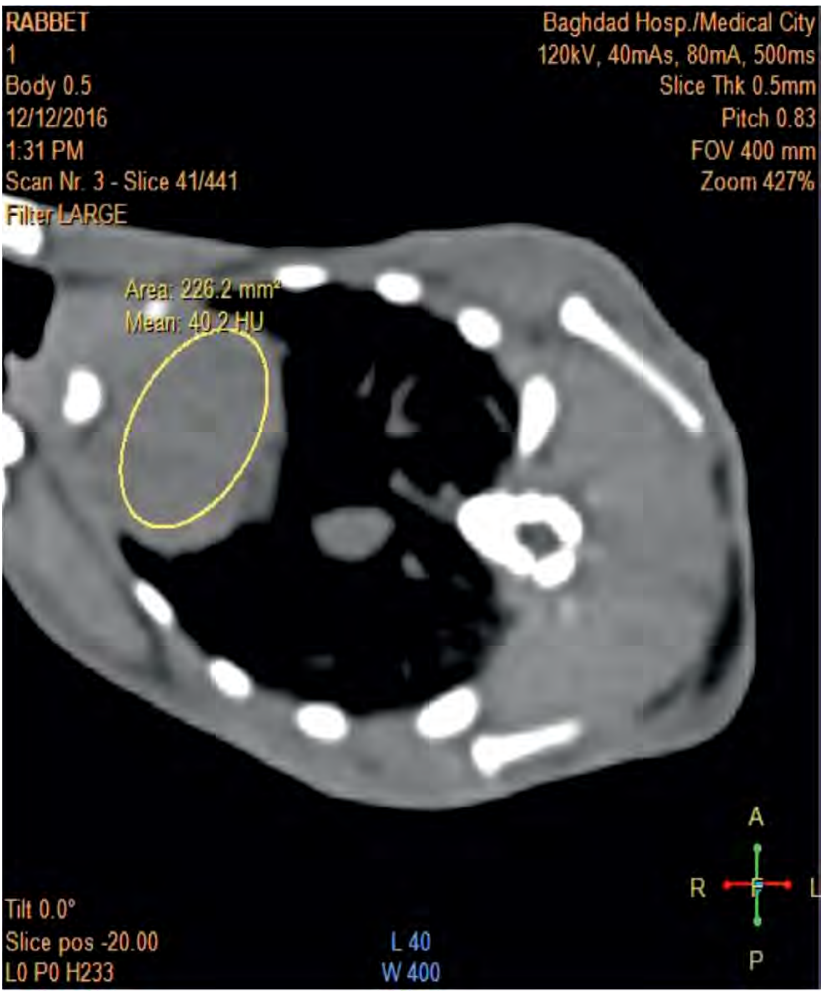

FIGURE 5. Illustrated CT-scan imaging of rabbit's heart native (without contrast medium) $(H U=40.2)$

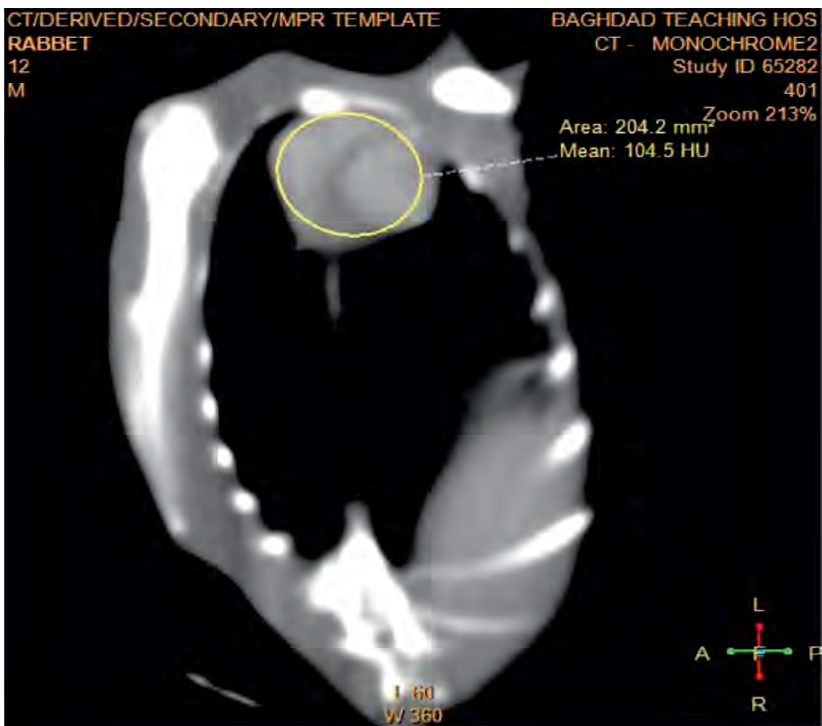

FIGURE 6. Illustrated CT-scan imaging of rabbit's heart using iodine contrast medium (HU=104.5)

\section{REFERENCES}

1. Radhi MM, Al-Shimmari HAT, Al-Mulla EAJ et al. New voltammetric study of $\mathrm{MgCl} 2$ as alternative contrast media in MRI molecular imaging. Nano Biomed Eng. 2017;9(2):152-161.

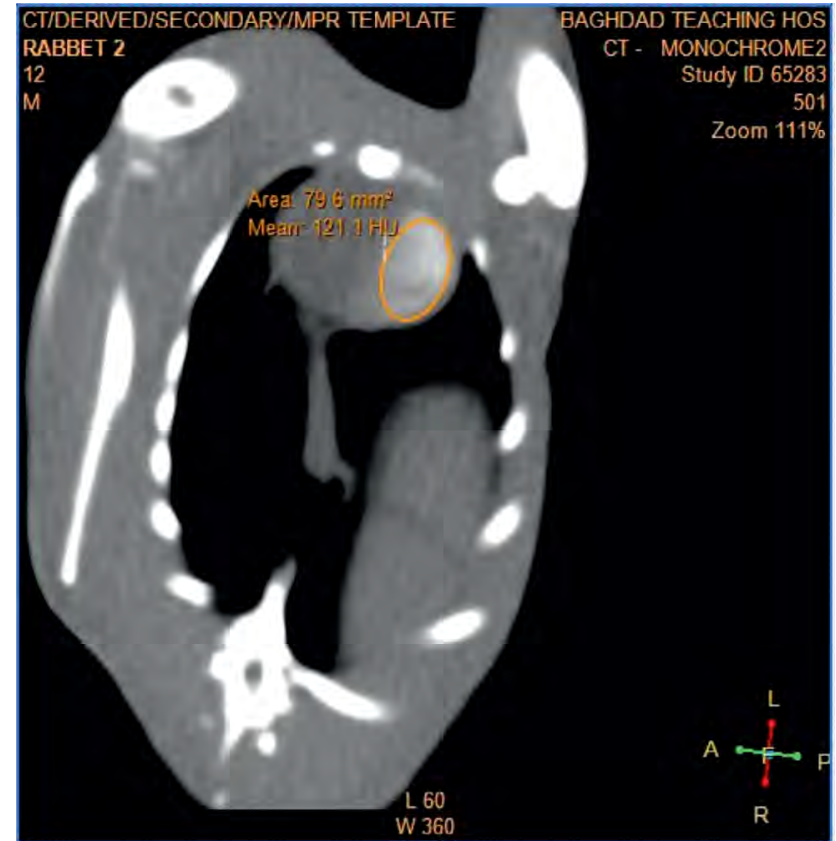

FIGURE 7. Illustrated CT-scan imaging of rabbit's heart using alternative contrast medium $\mathrm{MgSO}_{4}(\mathrm{HU}=121.1)$

\section{CONCLUSIONS}

Magnesium sulfate (MgSO4) solution has good electrochemical properties and safety for using as an alternative contrast medium without side effects for the patients of the CT-scan examination. $\mathrm{MgSO} 4$ solution has only a reduction current peak in blood medium, while the iodine compound has two oxidation current peaks, so magnesium sulfate is anti-oxidative compound and iodine is oxidative compound. On the other hand, $\mathrm{MgSO} 4$ solution has been used as an alternative contrast medium of iodine compound because the enhancement of imaging for the diagnosis of abdomen organs as found in the CT-scan of rabbit's heart which has HU $=121.1$ when using the alternative contrast and the value of $\mathrm{HU}$ is 104.5 when using iodine compound. We note the clear difference in the clarity of the CT-scan imaging and the value of HU. Furthermore, it can be noted the health safety of the alternative contrast medium (MgSO4).

Conflict of interest: none declared Financial support: none declared
2. Dunya Ali Mustafa, Haydar Abdulkadeer Taheer Al-Shimmari, Muhammed Mizher Radhi. Use of $\mathrm{MgCl} 2$ Nanoparticles as Alternative Contrast Media in Magnetic Resonance Imaging Molecular Imaging 
and Analyzed by Voltammetric Technique. Nano Biomed Eng. 2020;12(2):148-152.

3. Guidelines for Contrast Media from the European Society of Urogenital Radiology. AJR. 2003;181:1463-1471.

4. McDonald R, McDonald JS, Carter RE, Hartman RP, Katzberg RW et al. Intravenous Contrast Material Exposure Is Not an Independent Risk Factor for Dialysis or Mortality. Radiology. 2014;273(3):714-725.

5. Lee SY, Rhee CM, Leung AM, Braverman LE, Brent GA, Pearce EN. A Review: Radiographic lodinated Contrast Media-Induced Thyroid Dysfunction. J Clin Endocrinol Metab. 2014;100(2):376-83.

6. World Health Organization (2009). Stuart MC, Kouimtzi M, Hill SR (eds.). WHO Model Formulary 2008. World Health Organization. p. 316.

7. Karl Heinz Büchel, Hans-Heinrich Moretto, Dietmar Werner. Industrial Inorganic Chemistry. John Wiley \& Sons, 2d edition, 2000.

8. Nadolski GJ, Stavropoulos SW. Contrast alternatives for iodinated contrast allergy and renal dysfunction: Options and limitations. Journal of Vascular Surgery 2013;57(2):593-598.
9. Toon Van Cauteren, Gert Van Gompel, Koenraad H. Nieboer, Inneke Willekens, Paul Evans, Sven Macholl, Steven Droogmans. Improved enhancement in CT angiography with reduced contrast media iodine concentrations at constant iodine dose. Scientific Reports 2018;8:17493

10. Manouchehr Saljoughian. Intravenous Radiocontrast Media: A Review of Allergic Reactions. US Pharm. 2012;37(5):14-16.

11. Radhi MM, Al-Mulla EAJ. Use of a grafted polymer electrode to study mercury ions by cyclic voltammetry. Res Chem Intermed. 2015;41:1413-1420.

12. Boehm I. Is interleukin-2 (still) a risk factor for adverse reactions in concert with radiographic contrast medium injection. Acta Radiol. 2009;50(7):752-3.

13. Barrett BJ, Parfrey PS. Clinical practice. Preventing nephropathy induced by contrast medium. N Engl J Med. 2006;354(4):379-86.

14. Yildiz S, Kuşkonmaz SM. Effect of iodinated contrast media on thyroid: A brief review. Journal of Health Sciences 2016;6(1):12. 